\title{
A Review of Dietary Intake during Postpartum Period
} Jusoh NN, Tengku Ismail TA

Department of Community Medicine, School of Medical Sciences, University Sains Malaysia

\begin{abstract}
The postpartum period is a critical period for maternal and infant health. There is clear evidence showing the short- and long-term health benefits for women to adopt a healthy diet during this period. An unhealthy diet during this period can lead to cardiovascular disease and mental health problem among the mothers.

Keywords

dietary intake, nutrition, postpartum

Corresponding Author

Dr. Nur Nabila Jusoh

Department of Community Medicine,

School of Medical Sciences,

University Sains Malaysia.

Tel No: +6018-7893210

Email : nabilajusoh789@gmail.com

Received: 15 th July 2021; Accepted: 24th September 2021

Doi: https://doi.org/10.31436/imjm.v21i1

Besides, optimal food consumption during postpartum is crucial to support the additional nutrient requirements for breastfeeding and to reduce postpartum weight retention. Lack of social support and a harmful traditional dietary practices are among the barriers which prevent postpartum women from consuming balanced and nutritious food. Therefore, there is a need for effective nutritional intervention to promote better health for this population. In addition, the intervention should be culturally sensitive to respect the local traditional dietary practices for a better community acceptance.
\end{abstract}

\section{INTRODUCTION}

Childbirth is a memorable moment in every woman's life. It involves massive changes in her physical, physiological, psychological and social role. ${ }^{1}$ Even though the postpartum period can last up to 6 months after childbirth, some changes may take longer to be restored and some may never fully return to pre-pregnancy state. ${ }^{2}$

Dietary intake during this period poses short- and longterm health effects on both women and children. Unhealthy diet during postpartum put women in reproductive age at risk of cardiovascular disease ${ }^{3}$ and it also has impact on their mental health ${ }^{4,5}$. Besides, optimal food consumption during postpartum is essential to support the additional nutrient requirements for breastfeeding and to reduce postpartum weight retention. ${ }^{6}$

Aside from the aforementioned public health concerns, women are also anxious to return to their pre-pregnancy body weight and shape. ${ }^{7}$ They need evidence-based information on diet and nutrition to restore their body to the pre-pregnant state. It can prevent them from using improper weight loss method that claims speedy effect. ${ }^{8}$
Unfortunately, most women are unable to sustain a healthy diet that they have developed during pregnancy period, even though they understand the importance of postpartum nutrition.7, 9

They face barriers in maintaining healthy eating behaviour which can put them at risk of being overweight or obese post-pregnancy. Lack of social support from partners, families and friends is one of the barriers for them to adopt healthy eating practices. ${ }^{9}$ Besides, some longstanding traditional dietary practices can hinder postpartum women from consuming nutritious food, despite the higher energy and nutritional requirement during this period. ${ }^{10}$

This review aims to give an overview on dietary intake during the postpartum period. It hopes to generate information that enable various stakeholders to design more effective public health strategies to support women in adhering to healthier dietary behaviours throughout the postpartum period. 


\section{METHODS}

This narrative review was conducted by searching online databases including Google Scholar, PubMed and BMJ for articles and review papers using keywords of "dietary intake", "nutrition" and "postpartum". The inclusion criteria were articles published between 2000 - 2021, published reports, available full text articles and studies conducted among postpartum women. The articles published in languages other than English were excluded. Titles and abstracts screening were done based on the inclusion and exclusion criteria and the relevancy to the objectives of this review. Then, a total 47 articles were included in this review after the authors thoroughly checked the full articles. Five themes emerged from the chosen articles which include weight retention, traditional postpartum dietary practice, psychosocial support, mental health problem and breastfeeding.

\section{RESULTS}

\section{Macronutrient and micronutrient}

Generally, there is an increment of total fat intake at 6 months postpartum compared to pregnancy period, regardless of its measurement as an absolute intake or a proportion of energy..$^{11} \mathrm{It}$ is related to the poor diet quality after delivery, mostly due to high intake of nutrient-poor and energy dense foods such as cakes, ice cream and cookies. ${ }^{12}$ However, there is no consistency of evidence regarding changes in protein and carbohydrate intake from the pregnancy to the postpartum period. ${ }^{11}$

Studies in the United Kingdom showed that the micronutrient intake during pregnancy was not significantly different from the postpartum period. ${ }^{13}$ On the contrary, another study found that the intake of micronutrients including vitamins $\mathrm{A}, \mathrm{B}, \mathrm{C}, \mathrm{D}$, iron, zinc and calcium reduced from the third semester to postpartum period. ${ }^{12}$ The reduction can be explained by the decrease in vegetables and fruit intakes which are the primary source of micronutrients. Besides, there is also a decline in supplement use among women after childbirth. ${ }^{12}$
In terms of supplementation during the postpartum period, despite the lack of evidence from randomized control trials, the World Health Organisation recommended iron and folic acid supplements for at least 3 months postpartum. ${ }^{14}$ It is based on the high anaemia prevalence during postpartum, especially in countries with high maternal mortality rate. The report ${ }^{14}$ acknowledged that there are side effects from the oral iron supplement, but they are not life-threatening.

\section{Weight retention}

Generally, postpartum weight retention is defined as the difference between pre-pregnancy weight and weight after delivery. ${ }^{15}$ Postpartum weight retention is a concern because it contributes to the long-term development of obesity among women in reproductive age and interpregnancy weight gain. ${ }^{3}$ In addition, there is a causal relationship between high postpartum weight retention with gestational diabetes, stillbirth and large-forgestational-age infant in the subsequent pregnancies. ${ }^{16}$

A systematic review estimated that the mean weight retention among five Asian countries was between 1.56 $4.1 \mathrm{~kg}$ at 6 months postpartum, with the Chinese showed the highest weight retention. ${ }^{17}$ In terms of prevalence, $33.8 \%$ of Malaysian women retained more than $5 \mathrm{~kg}$ at 6 months postpartum. ${ }^{1}$ Whereas in the West, a study in Amsterdam discovered that one in five women had a weight gain of more than $5 \mathrm{~kg}$ at one year postpartum. ${ }^{18}$

The link between dietary intake and postpartum weight retention was previously established. Women who exceed the local dietary recommendation of saturated fatty acid had a more than threefold higher odds ( $\mathrm{OR}=3.40,95 \%$ CI: 1.04 - 11.11) to retain more than $5 \mathrm{~kg}$ at one year postpartum compared with others. ${ }^{18}$ High energy intake in diet was associated with weight retention of more than $5 \mathrm{~kg}$ at 6 months postpartum among Malaysian women. ${ }^{8}$ However, multivariable analysis in the study did not find significant associations between postpartum weight retention and protein, carbohydrate or fat intake. The same study also found that breastfeeding for at least 6 months reduces postpartum weight retention ${ }^{8}$ because 
women burn approximately $500 \mathrm{kcal}$ per day while breastfeeding. ${ }^{19}$

A cohort study in the United States showed that total energy intake positively associated with postpartum weight retention. ${ }^{20}$ Instead, the dietary pattern based on the Mediterranean Diet Score and the Alternative Healthy Eating Index-2020 was not associated with postpartum weight retention. Thus, it suggested that quality of food consumption have a minor role compared to its quantity.

Nonetheless, another study in Lebanon and Qatar showed that a high intake of proteins, trans fat, cholesterol, sodium and lower intakes of polyunsaturated fat were linked to a greater postpartum weight retention when adjusted to energy intake. ${ }^{21}$

A multi-ethnic cohort study 22 among antenatal women in Norway discovered that women with ethnic origin from South Asia, Middle East and Africa had significantly higher postpartum weight retention than Western European women. Notably, an unhealthy diet was not associated with weight retention. ${ }^{22}$ Perhaps, the ethnic groups altered their traditional diet to an unhealthier Western diet after migration.

Besides, hormone also plays a role in determining the postpartum weight retention. Plasma leptin has been suggested as a predictor of postpartum weight retention. The concentration of leptin increases with increasing adiposity. ${ }^{23}$ A cohort study among 545 women discovered that South Asians who have higher plasma leptin in early pregnancy, retained more weight and subcutaneous fat at 14 weeks postpartum compared to the Europeans. ${ }^{24}$ Besides, the South Asians also have slower decline of plasma leptin than the Europeans. On the other hand, a study among Korean women showed that gestational plasma leptin level is not significantly associated with postpartum weight retention. ${ }^{25}$ This discrepancy may be due to small number of sample size $(n=75)$ in the Korean study. ${ }^{25}$

In terms of intervention, a meta-analysis showed that nutritional intervention during postpartum had a significantly greater weight reduction compared to usual standard care. ${ }^{26}$ It was found that characteristics of effective intervention include monitor weight regularly, combine both diet and physical activity in the intervention and the intervention is delivered by health professionals. ${ }^{26}$, 27 Surprisingly, the magnitude of weight loss was not affected by intervention intensity (duration and number of sessions) and setting (individual or group). ${ }^{27}$ The evidence of the characteristics of an effective nutritional intervention can help policymakers in developing intervention at their local setting.

In addition, intervention during the postpartum period should consider women's acceptability. A qualitative study on postpartum women who had pre-pregnancy BMI $\geq 30$ $\mathrm{kg} / \mathrm{m}^{2}$ observed that they were aware that the initiative to change lifestyle should come from them. ${ }^{7}$ However, they perceived that eating habits and weight is not a central issue at that point in their lives. Their focus was more on the new routine of baby care.

\section{Traditional postpartum dietary practice}

Cultural influences also play an essential role in determining postpartum diet. This area was explored extensively by various studies, ${ }^{10,28-32}$ this article will only give a general overview of the findings.

In non-Western culture, the emphasise is on the women themselves and social support rituals by family members (enthnokinship) for an extended period. ${ }^{28}$ During this period, the women receive extra care and they are prohibited from doing household chores. Therefore, the non-Western culture is collectivistic while Western culture is more individualistic. ${ }^{29}$

Most traditional dietary practices are linked to nonWestern cultures. The traditional dietary practices are distinct in between countries, but they share few similarities. Women in the non-Western culture are prescribed a period of rest which they are prohibited from doing household chores and need to adhere to certain traditional dietary practices. ${ }^{30}$ In a systematic review of publications from 20 countries, the postpartum period 
ranges from 21-35 days. ${ }^{30}$ A 40-day rest period is similarly practised in countries in various countries such as Mexico, Malaysia, South Africa, and Japan. ${ }^{30}$

However, the length of the rest period is also determined by socio-economic status. Even though most Cambodian women had a rest period for 2-3 months, women from the high socioeconomic group had extended rest period because they can afford to reduce their normal activities for a longer time. ${ }^{31}$

The concept behind the traditional dietary practice is that the local food is categorised into 'hot' and 'cold' regardless of the food's real temperature. ${ }^{30}$ Different cultures have its unique classification, as shown by the comprehensive summary on food taboos during the postpartum period in Southeast Asia countries. ${ }^{10,} 32$ Since the postpartum period is considered as cold and vulnerable, consuming 'hot' food will counter by restoring the balance. ${ }^{30}$

Adhering to the traditional dietary practice is believed to avoid immediate ill-health on both postpartum women (e.g. postpartum haemorrhage, swollen and watery uterus, fatigue or digestive disorders) and newborn (e.g. lost consciousness and late umbilical cord dryness). ${ }^{30}$ Nonadherence to this practice can also cause illness in later life. For instance, some women regret in later life when they experience multiple joint pain due to the nonadherence to these traditional practices. ${ }^{30}$ The traditional practice is also intended to restore sexual and reproductive function, boost mothers' energy, escalate wound healing and return to pre-pregnancy weight. ${ }^{29}$

\section{Psychosocial support}

In non-Western cultures, postpartum women receive social support from mothers, mothers-in-law, female relatives, traditional birth attendants or husbands. Women who are from the middle or upper socio-economic class can hire people to provide necessary support. Female predominantly provide the social support because they have more experience and men have fear of contamination. ${ }^{30}$
Social support not only helps postpartum women to prepare meals but it also boost their self-efficacy in which they believe that they have the capacity to make healthy choices in their daily lives. ${ }^{33}$ With increasing self-efficacy, individuals expect positive outcomes, overcome barriers and be motivated to achieve their purpose. ${ }^{34}$

In a conceptual model proposed by Phillips et al. (2012), social support was also acknowledged as one of the predictors for maternal behaviour such as dietary quality, breastfeeding and physical activity. ${ }^{35}$ Subsequently, these behaviours will affect the maternal weight post-delivery.

A study on 1356 postpartum women in the United States found that high support from partners was associated with high fibre and low trans-fat intake among these women. 36 This finding suggests that partners may assist the new mothers in the activity daily living such as buying groceries, preparing healthy food and eating healthy food together. However, the PrimeScreen dietary survey used in this study did not assess the total diet to measure absolute nutrient intake. In addition, this study revealed that social support from the partner, families and friends was also related to high physical activity.

A qualitative study showed that postpartum women felt helpless, lonely and have no self-care time as they focus on infant care. They use food to relieve displease as a compensatory mechanism because they are unable to evaporate their feeling adequately. Hence, family members need to support them by helping them with the new routine to avoid this unhealthy eating behaviour. ${ }^{7}$

\section{Mental health problem}

Nutrition also plays a role to prevent postpartum depression. Dietary fat is not solely for energy source but also has an impact on brain function. Intake of polyunsaturated fatty acids (PUFAs) including docosahexaenoic acid (DHA) has a prominent role in receptor function, membrane fluidity, neurotransmitter uptake and signal transmission. ${ }^{37}$ The PUFAs insufficiency can affect the serotonin neurotransmitter, which is associated with depression pathophysiology. ${ }^{37}$ 
Since human have limited ability to synthesis long-chain PUFAs, they should be consumed from various sources. They can be found in high concentration in aquatics (mackerel, tone and code), animal sources (fish, meat, egg and milk), plant sources (herbs, spices and fruit seeds). ${ }^{38}$ Besides, nutrients such as folate and vitamin-B12 is also associated with postpartum depression. ${ }^{37}$ Thus, nutritional intervention may have a potential to prevent and treat postpartum depression.

Teo et al. (2018) showed that the traditional Indian dietary pattern had a protective effect against postpartum, which might be explained by the high vitamin-B in Indian food such as legumes and ethnic bread. ${ }^{5}$ Furthermore, the soup -vegetable-fruit dietary pattern was associated with less postpartum anxiety symptoms. ${ }^{5}$ It is most likely because vegetables and fruits which are rich in antioxidants, can help to counter balance the effect of free radical oxidants and provide neural protection. ${ }^{39}$

A study in China on 924 postpartum women showed that high food diversity (more than 6 types of food) had a negative association with depression status. The possible explanation was food variety can achieve the adequacy of nutrients to reduce the risk of depression. ${ }^{4}$ Unfortunately, strict adherence to the traditional postpartum food restrictions may reduce their food choice and food diversity. Subsequently, this will put them at higher risk of depression.

\section{Breastfeeding}

The energy and nutritional requirement during breastfeeding differ from the general population. Breastfeeding women need approximately 500 additional $\mathrm{kcal} /$ day compared to non-pregnant women. ${ }^{19}$ However, maternal calorie, macronutrients, dietary fibre and water intake during the postpartum period do not meet the recommended values. ${ }^{19}$ It can be attributed to the restriction of food types and quantity among those who practise the traditional confinement. ${ }^{19}$ The levels of fatand water-soluble vitamins in breast milk are reduced when there is a maternal vitamin deficiency. However, calcium, phosphorus and magnesium levels in breast milk are unrelated to the maternal level and diet. ${ }^{40}$
Breastfeeding during the postpartum period has impact on postpartum weight retention. A study among Malaysian women discovered that continuing breastfeeding until six months had significant lower postpartum weight retention. ${ }^{8}$ Another study showed that the longer the breastfeeding period, the lower the postpartum weight retention. ${ }^{41}$ Among those who breastfeed for 6 months, every month of breastfeeding will contribute to $0.44 \mathrm{~kg}$ of weight loss compared to only a month of breastfeeding. It can be explained by the usage of maternal body stores if they do not take additional calories during breastfeeding. ${ }^{40}$

Traditional practice during the postpartum period also influences nutrition among lactating mothers. There is a traditional belief that specific food intake may change breastmilk taste. For example, women in rural Indonesia believe that eating fish can make breastmilk to have an unpleasant smell and bad taste. ${ }^{42}$ However, baby rarely reacts to the food consumed by the mothers; thus it is not justified to avoid particular foods during breastfeeding. ${ }^{43}$

Certain traditional dietary practice during postpartum can be harmful to breastfed children, leading to morbidity and mortality. For example, in Laos, postpartum women restrict their diet to long soaked polished glutinous rice. ${ }^{44}$ It results in maternal thiamine deficiency and low thiamine in breastmilk. Consequently, the fully breastfed infant are at higher risk of infantile thiamine deficiency which can cause severe heart failure. ${ }^{44}$

In Laos, an evaluation of interventions to reduce the traditional food restriction among postpartum women was conducted. ${ }^{45}$ It showed that women showed positive dietary changes if village health volunteers disseminate correct information and community members participate in planning, implementing and evaluating the program. In addition, the message conveyed via mass media is also effective, especially when it is consistent with the government policy (i.e. food restriction during the postpartum period is harmful and food diversity is recommended) .45

Intervention to alter harmful traditional practice is challenging. The first step is to train healthcare 
professional with evidence-based recommendations regarding dietary intake during postpartum. ${ }^{46}$ It is because health facility attendance can be a golden opportunity for nutritional education to the mothers. A survey was conducted on Thai nurses who worked at antenatal clinic, delivery room, postpartum ward and community to explore nurses' perspective of postpartum practices. Despite recommending a balanced diet of nutritious food to patients, they also emphasize several foods restriction. ${ }^{47}$ Approximately one in four of the nurses agreed that postpartum women should practice the traditional food restriction. ${ }^{47}$

\section{CONCLUSION}

Postpartum dietary intake has been to influence maternal and child health. An unhealthy diet during this period can lead to cardiovascular disease and mental health problem among the mothers, and malnutrition of the infants. Besides, a balanced and nutritious diet is essential to support breastfeeding practice. Despite higher diet requirement, lack of social support and some harmful traditional dietary practices are the barriers which hinder postpartum women from getting optimal nutrition. The postpartum period offers a golden opportunity for health education and health promotion. Therefore, policymakers should plan for effective nutritional intervention because it is a high return investment for future community health. However, for better acceptance by the community, the intervention should be culturally sensitive to respect the local tradition and consider emotional fragility during this period.

\section{CONFLICTS OF INTEREST}

The authors declare no conflict of interest.

\section{REFERENCES}

1. Fadzil F, Shamsuddin K, Wan Puteh SE. Traditional postpartum practices among Malaysian mothers: A review. J Altern Complement Med. 2016;22(7):503-8.

2. Romano M, Cacciatore A, Giordano R, La Rosa B. Postpartum period: three distinct but continuous phases. J Prenat Med. 2010;4(2):22-5.
3. Bogaerts A, Devlieger R, Van den Bergh BR, Witters I. Obesity and pregnancy, an epidemiological and intervention study from a psychosocial perspective. Facts Views Vis Obgyn. 2014;6(2):81-95.

4. Jiang W, Mo M, Li M, et al. The relationship of dietary diversity score with depression and anxiety among prenatal and post-partum women. J Obstet Gynaecol Res. 2018;44(10):1929-36.

5. Teo C, Chia AR, Colega MT, et al. Prospective associations of maternal dietary patterns and postpartum mental health in a multi-ethnic Asian cohort: The Growing up in Singapore towards Healthy Outcomes (GUSTO) Study. Nutrients. 2018;10(3).

6. Falciglia G, Piazza J, Ritcher E, Reinerman C, Lee SY. Nutrition education for postpartum women:A pilot study. J Prim Care Community Health. 2014;5 (4):275-8.

7. Faria-Schützer DB, Surita FG, Rodrigues L, Turato ER. Eating behaviors in postpartum: A qualitative study of women with obesity. Nutrients. 2018;10(7).

8. Fadzil F, Shamsuddin K, Wan Puteh SE, et al. Predictors of postpartum weight retention among urban Malaysian mothers: A prospective cohort study. Obes Res Clin Pract. 2018;12(6):493-9.

9. Kaiser B, Razurel C. Determinants of postpartum physical activity, dietary habits and weight loss after gestational diabetes mellitus. J Nurs Manag. 2013;21 (1):58-69.

10. Köhler R, Sae-tan S, Lambert C, Biesalski HK. Plantbased food taboos in pregnancy and the postpartum period in Southeast Asia - a systematic review of literature. Nutr Food Sci. 2018;48(6):949-61.

11. Lee YQ, Loh J, Ang RSE, Chong MF-F. Tracking of maternal diet from pregnancy to postpregnancy: A systematic review of observational studies. Current Developments in Nutrition. 2020;4(8).

12. Lebrun A, Plante AS, Savard C, et al. Tracking of dietary intake and diet quality from late pregnancy to the postpartum period. Nutrients. 2019;11(9).

13. Derbyshire E, Davies GJ, Costarelli V, Dettmar PW. Habitual micronutrient intake during and after pregnancy in Caucasian Londoners. Matern Child Nutr. 2009;5(1):1-9. 
14. World Health Organisation. Recommendations on postnatal care of the mother and newborn 2014

[Available from: https://apps.who.int/iris/ handle/10665/97603.

15. Oken E, Taveras EM, Popoola FA, Rich-Edwards JW, Gillman MW. Television, walking, and diet: associations with postpartum weight retention. Am J Prev Med. 2007;32(4):305-11.

16. Villamor E, Cnattingius S. Interpregnancy weight change and risk of adverse pregnancy outcomes: a population-based study. Lancet. 2006;368 (9542):1164-70.

17. Cheng HR, Walker LO, Tseng YF, Lin PC. Postpartum weight retention in women in Asia: a systematic review. Obes Rev. 2011;12(10):770-80.

18. Althuizen E, van Poppel MNM, de Vries JH, Seidell JC, van Mechelen W. Postpartum behaviour as predictor of weight change from before pregnancy to one year postpartum. BMC Public Health. 2011;11(1):165.

19. Munirah S, Abdul Basir SM, Radiah A, et al. Dietary status of exclusively breastfeeding mothers: The influence of traditional postpartum dietary practices. Int J Health Allied Sci. 2020;3(2):634-42.

20. Boghossian NS, Yeung EH, Lipsky LM, Poon AK, Albert PS. Dietary patterns in association with postpartum weight retention. Am J Clin Nutr. 2013;97(6):1338-45.

21. Nasreddine L, Ayoub J, Abbas N, Malik M, Naja F. Postpartum weight retention and its determinants in Lebanon and Qatar: Results of the Mother and Infant Nutrition Assessment (MINA) Cohort. Int J Environ Res Public Health. 2020;17:7851.

22. Waage C, Falk R, Sommer C, et al. Ethnic differences in postpartum weight retention: a Norwegian cohort study. BJOG: An International Journal of Obstetrics \& Gynaecology. 2016;123 (5):699-708.

23. Sámano R, Martínez-Rojano $\mathrm{H}$, Chico-Barba $\mathrm{G}$, et al. Serum Concentration of Leptin in Pregnant Adolescents Correlated with Gestational Weight Gain, Postpartum Weight Retention and Newborn Weight/Length. Nutrients. 2017;9(10).
24. Sommer C, Jenum AK, Waage CW, et al. Ethnic differences in BMI, subcutaneous fat, and serum leptin levels during and after pregnancy and risk of gestational diabetes. Eur J Endocrinol. 2015;172 (6):649-56.

25. Kim KH, Kim YJ, Lee S, et al. Evaluation of plasma leptin levels \& BMI as predictor of postpartum weight retention. Indian J Med Res. 2008;128(5):595 -600 .

26. Vincze L, Rollo M, Hutchesson M, et al. Interventions including a nutrition component aimed at managing gestational weight gain or postpartum weight retention: a systematic review and meta-analysis. JBI Database System Rev Implement Rep. 2019;17(3):297-364.

27. Lim S, Liang X, Hill B, et al. A systematic review and meta-analysis of intervention characteristics in postpartum weight management using the TIDieR framework: A summary of evidence to inform implementation. Obes Rev. 2019;20(7):1045-56.

28. Posmontier B, Horowitz JA. Postpartum practices and depression prevalences: technocentric and ethnokinship cultural perspectives. J Transcult Nurs. 2004;15(1):34-43.

29. Hishamshah M, Ammar S, Khan A, et al. Belief and practices of traditional post partum care among a rural community in Penang Malaysia. Int J Third World Med. 2010;9(2).

30. Dennis CL, Fung K, Grigoriadis S, et al. Traditional postpartum practices and rituals: a qualitative systematic review. Womens Health (Lond). 2007;3 (4):487-502.

31. White PM. Heat, balance, humors, and ghosts: postpartum in Cambodia. Health Care Women Int. 2004;25(2):179-94.

32. Köhler R, Lambert C, Biesalski HK. Animal-based food taboos during pregnancy and the postpartum period of Southeast Asian women - A review of literature. Food Res Int. 2019;115:480-6.

33. Sheeran P, Maki A, Montanaro E, et al. The impact of changing attitudes, norms, and self-efficacy on health-related intentions and behavior: A metaanalysis. Health Psychol. 2016;35(11):1178-88. 
34. Bandura A. Health promotion by social cognitive means. Health Educ Behav. 2004;31(2):143-64.

35. Phillips J, King R, Skouteris H. A conceptual model of psychological predictors of postpartum weight retention. J Reprod Infant Psychol. 2012;30:1-11.

36. Faleschini S, Millar L, Rifas-Shiman SL, et al. Women's perceived social support: associations with postpartum weight retention, health behaviors and depressive symptoms. BMC Women's Health. 2019;19(1):143.

37. Rechenberg K, Humphries D. Nutritional interventions in depression and perinatal depression. Yale J Biol Med. 2013;86(2):127-37.

38. Abedi E, Sahari MA. Long-chain polyunsaturated fatty acid sources and evaluation of their nutritional and functional properties. Food Sci Nutr. 2014;2 (5):443-63.

39. Scapagnini G, Davinelli S, Drago F, De Lorenzo A, Oriani G. Antioxidants as Antidepressants. CNS drugs. 2012;26:477-90.

40. Kominiarek MA, Rajan P. Nutrition recommendations in pregnancy and lactation. Med Clin North Am. 2016;100(6):1199-215.

41. Kac G, Benício MH, Velásquez-Meléndez G, Valente JG, Struchiner CJ. Breastfeeding and postpartum weight retention in a cohort of Brazilian women. Am J Clin Nutr. 2004;79(3):487-93.

42. Agus Y, Horiuchi S, Porter SE. Rural Indonesia women's traditional beliefs about antenatal care. BMC Res Notes. 2012;5:589.

43. Jeong G, Park SW, Lee YK, Ko SY, Shin SM. Maternal food restrictions during breastfeeding. Korean J Pediatr. 2017;60(3):70-6.

44. Barennes H, Sengkhamyong K, René J, Phimmasane $\mathrm{M}$. Beriberi (thiamine deficiency) and high infant mortality in Northern Laos. PLoS Negl Trop Dis. 2015;9:e0003581.

45. Ayano K, Ohnishi M. An evaluation of strategies to promote health to tackle food restriction in postpartum women in Lao People's Democratic Republic. Health Sci Res. 2017;29:59-66.
46. Barennes H, Simmala C, Odermatt $P$, et al. Postpartum traditions and nutrition practices among urban Lao women and their infants in Vientiane, Lao PDR. Eur J Clin Nutr. 2009;63(3):323-31.

47. Kaewsarn P, Moyle W, Creedy D. Thai nurses' beliefs about breastfeeding and postpartum practices. J Clin Nurs. 2003;12(4):467-75. 